\title{
Effekte einer abrupten Landnutzungsintensivierung auf den Feldgrashüpfer (Chorthippus apricarius) zum Laufzeitende einer extensiven Großweidenutzung
}

\author{
Effects of a sudden land-use intensification on the Locomotive Gras- \\ shopper (Chorthippus apricarius) in a former large pasture landscape
}

\author{
Henning Nissen ${ }^{(D)}$ \\ Institut für Natur- und Ressourcenschutz, Abteilung für Landschaftsökologie, Christian-Albrechts-Universität zu Kiel, \\ Olshausenstr. 75, 24118 Kiel, hnissen@ecology.uni-kiel.de
}

\section{Schlüsselwörter: \\ Orthoptera, Großweideland- schaft, Populationsrückgang, Intensivierung}

\begin{abstract}
Zusammenfassung
Durch eine Nutzungsintensivierung mit einhergehender Abnahme der Strukturvielfalt der seit 2000 extensiv beweideten Großweidelandschaft im Oberen Eidertal hat sich die lokale Population des Feldgrashüpfers (Chortippus apricarius) räumlich verschoben und ist in weiten Teilen der ehemaligen zusammenhängenden Flächen stark zurückgegangen. Während die kontinuierliche Nutzung als Großweidelandschaft (1999-2018) keinen negativen Effekt auf die Bestände des Feldgrashüpfers hatte, so wurden nach der Intensivierung 2019 viele Teilbereiche der Weideflächen nicht mehr besiedelt. Grundlage der Untersuchung bildet einen Vergleich dreier räumlich und methodisch identischer Kartierungen in 1999/2000, 2018 und nach der Intensivierung in 2019.
\end{abstract}

\begin{abstract}
The intensification of a large pasture landscape in the Upper Eider-valley and thus, the decrease of the structural diversity, led to a local spatial shift and a population decline of the Locomotive Grasshopper (Chorthippus apricarius). Since 2000, the pasture landscape was ecologically managed by moderate cattle grazing. No negative effect on the populated area of $C$. apricarius was observed during the continuous large pasture landscape management (1999-2018), in 2019 intensification led to an immediate decline of these areas. Three similar investigations were conducted in 1999/2000, 2018 and after landuse intensification 2019, and the results are compared.
\end{abstract}

\section{Einleitung}

Der Feldgrashüpfer ist in weiten Teilen Europas und Asiens verbreitet und hat seinen Verbreitungsschwerpunkt in den zentralasiatischen Steppen. In Deutschland ist der Feldgrashüpfer mit Ausnahme des Saarlandes aus allen Bundesländern bekannt, zum Teil beschränken sich die Vorkommen jedoch auf sehr kleine Flächen oder sehr lokale Vorkommen (Reck 1998). Obwohl die Art in Deutschland nicht gefährdet ist
(Maas et al. 2011), gilt sie u.a. in Baden-Württemberg als vom Aussterben bedroht (Detzel 1998) und Bayern als stark gefährdet (Voith et al. 2016), in SchleswigHolstein ist die Art nicht gefährdet (Winkler \& Klinge 2019, Winkler \& Haacks 2019). Hier ist der Feldgrashüpfer v.a. in der Geest weit verbreitet (Abb. 1).

Trotz weiter Verbreitung kommt es infolge von anhaltender Intensivierung der Landwirtschaft, Eutrophierung, Flurbereinigung und der nicht naturschutzfachlichen Pflege von Straßenbegleitflächen zu lokalen Rückgängen 
und dem Erlöschen kleiner Restpopulationen (Buchweitz et al. 1990, Reck 2004, Illich 2017, Worschech 2017).

Der Feldgrashüpfer bevorzugt insbesondere Feldraine zwischen Äckern und Bereiche mit heterogener Vegetationsstrukturen auf mineralischen Grünlandflächen. Vor allem Lebensräume an Nutzungsübergängen und -grenzen werden besiedelt (Buchweitz et al. 1990, Reck 1998, Winkler 1999, Köhler 2001, Schulz 2003a, Reck 2004). Nicht nur die o.g. Strukturen, sondern auch bestimmte Mikrohabitate (z.B. kleine Störstellen in der Grasnarbe, Tierbauten) stellen für den Feldgrashüpfer wichtige, teils sogar essenzielle Habitatbausteine in unterschiedlichen Stadien seines Lebenszyklus dar (Reck 1993, Schulz 2003b, Worschech 2017).

Im Modellprojekt ,Weidelandschaft Eidertal ‘ wurden zwischen ca. 1999 und 2018 auf ca. 400 ha intensiv genutzte Ackerflächen und verschiedene Grünlandtypen zu großflächigen extensiven Weidesystemen zusammengefasst (Jensen et al. 2001). Nach dem Auslaufen der 20jährigen Vertragsnaturschutzlaufzeit wurden in 2018 erste Teile aus diesem großflächigen Weidesystem herausgenommen, damit kleiner parzelliert und intensiver und homogener genutzt. Der Effekt dieser Intensivierung und der damit verbundene Rückgang des Feldgrashüpfers auf diesen Flächen soll durch den Vergleich der Ergebnisse dreier Kartierungen in unterschiedlichen Jahren beschrieben werden.

\section{Gebietsbeschreibung und Methoden}

Das Projektgebiet der „Weidelandschaft Eidertal“ erstreckt sich über etwa 400 ha im Talraum der Oberen Eider bei Flintbek im Naturraum „Östliches Hügelland“. Der Talraum ist durch meist entwässerte und heute in der Wiedervernässung befindliche Niedermoore gekennzeichnet. Diese gehen randlich zu quelligen Bereichen über, die vorwiegend als Feuchtweiden (Flutrasen) genutzt wurden oder sich infolge von Nutzungsaufgaben zu Feuchtgrünland-Brachen entwickelten (Jensen et al. 2001). Die umgebenden und in weiten Teilen in die Weidelandschaft einbezogenen Talhänge mit mineralischem Boden wurden vor der Extensivierung sowohl ackerbaulich, als auch als Grünland genutzt. Der untersuchte Teil der zusammenhängenden Weidelandschaft besteht seit 2000. Hier wurden Ackerflächen in Grünland umgewandelt und bestehendes Grünland extensiviert. Sie erstreckt sich über ca. 20 ha und ist durch eine hohe Strukturviel- falt charakterisiert (Abb. 1). Nasse Standorte mit Flutrasengesellschaften entlang des Flusses und in Senken werden durch Feuchtwiesen abgelöst. An den Hängen folgen mineralische Standorte mit trockenem Charakter auf den Kuppen. Als weitere Strukturelemente sind Knicks und Feldgehölze vorhanden (Abb. 2).

Die zusammenhängende Weide wurde knapp 20 Jahren lang im Rahmen einer Extensivbeweidung genutzt und dann Anfang 2019 erstmals wieder gemäht und Teile des Knicknetzes wurden auf den Stock gesetzt. Nach Abschluss dieser Arbeiten wurde die Weide neu parzelliert, nasse Flächen entlang der Eider ausgezäunt, der nördliche (Intensivierung 2019 / Nord; Rinder- und Schafsweide) mit ca. 3 ha und der südliche Bereich (Intensivierung 2019 / Süd; Rinderweide) mit 7,6 ha wurden mit einer höheren GVE beweidet, der ca. 1,5 ha große mittlere Teil lag brach (Tab. 1 \& Abb. 2 rechts). Vor Einrichtung des Weidesystems wurde die Dichte und Verbreitung der Feldgrashüpfer in 1999 (B. Holsten) und 2000 (B. Schulz) flächig kartiert. Eine erneute Kartierung erfolgte vom Verfasser in 2018 auf der dann noch bestehenden großflächigen Weide. Im August 2019, wenige Monate nach der Intensivierung, wurde die Fläche erneut kartiert.

Bei der Erfassung wurde die Dichte der Population an jeweils drei geeigneten Terminen (sonnig, Temp. $>25^{\circ} \mathrm{C}$ ) mit Hilfe der Verhörmethode, also anhand der Dichte stridulierender Männchen, abgeschätzt. Die Lage und Größe der kartierten Fläche war in allen Jahren identisch und wurde bis auf die nassen Bereiche vollständig und schleifenförmig begangen.

Die Individuen wurden gezählt bzw. deren Anzahl konservativ geschätzt. Die Anzahl wurde den folgenden, von Holsten und Schulz angewendeten Häufigkeitsklassen zugeordnet:

\begin{tabular}{|c|l|}
\hline I & Einzelne ơ je $100 \mathrm{~m}^{2}$. \\
\hline II & geringe Dichte $\left(1-10 \sigma^{\top} / 100 \mathrm{~m}^{2}\right)$ \\
\hline III & mittlere Dichte $\left(11-50 \mathrm{o}^{7} / 100 \mathrm{~m}^{2}\right)$ \\
\hline IV & hohe Dichte $\left(>50 \mathrm{o}^{7} / 100 \mathrm{~m}^{2}\right)$ \\
\hline
\end{tabular}

Einzeltiere wurden punktuell, die bei den Begehungen festgestellten flächigen Bestände wurden räumlich in der Karte abgegrenzt eingetragen. Anschließend wurden die von Hand eingetragenen Dichteklassen in ein Geografisches Informationssystem (ESRI ${ }^{\circledR}$ ArcMap 
Tab. 1: Nutzung und strukturelle Charakterisierung der untersuchten Flächen in den Jahren der Feldgrashüpferkartierungen.

\begin{tabular}{lllll} 
Jahr & Fläche & Nutzung & Nutztiere & Lebensraumrequisiten \\
\hline $\mathbf{1 9 9 9 / 2 0 0 0}$ & $\begin{array}{l}\text { Intensivierung 2019 Nord } \\
\text { Intensivierung 2019 Süd } \\
\text { Brache 2019 }\end{array}$ & Großweidelandschaft & Rinder & A, B, C, D \\
\hline $\mathbf{2 0 1 8}$ & $\begin{array}{l}\text { Intensivierung 2019 Nord } \\
\text { Intensivierung 2019 Süd }\end{array}$ & Großweidelandschaft & Rinder & A, B, C, D \\
& Brache 2019 & & \\
\hline $\mathbf{2 0 1 9}$ & Intensivierung 2019 Nord & Weide & & \\
& Intensivierung 2019 Süd & Weide & Rinder, Schafe & E, F \\
& Brache 2019 & Weidebrache & Rinder & E, F \\
& & & A, B, C
\end{tabular}

Erläuterungen: A: vielzählige, hochwüchsige Saumstrukturen; B: heterogene Knickstrukturen; C: vielfältige, kleinstrukturierte Grünlandlebensräume (unterschiedlicher Verbiss-, Vertritt- und Aufwuchsintensitäten); D: kleinflächige Hochstaudenfluren; E: homogene Weidefläche, nahezu keine andersartigen Mikrohabitate vorhanden; F: auf den Stock gesetzte Knicks.

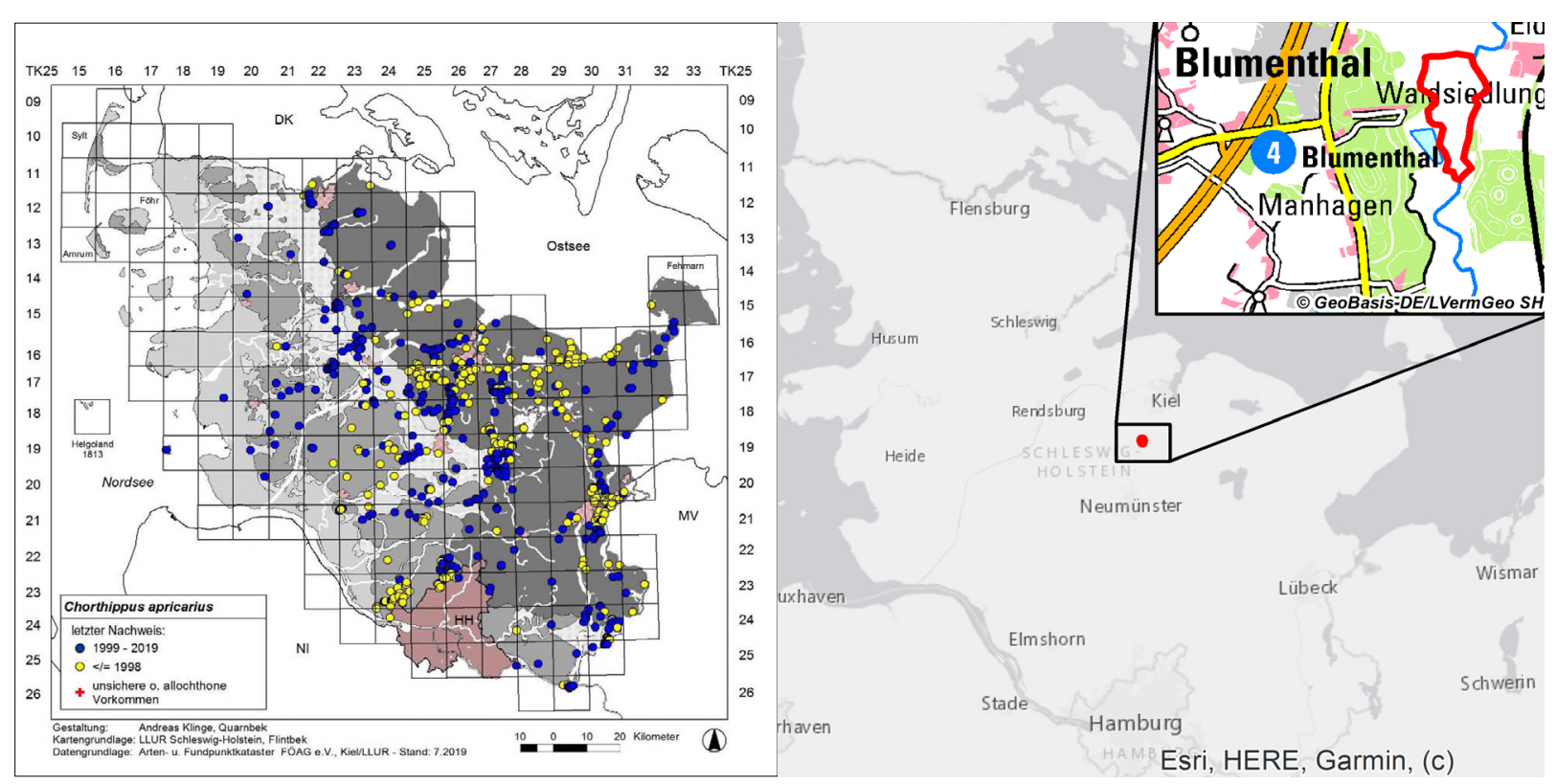

Abb. 1: Die aktuelle Verbreitung des Feldgrashüpfers (Chorthippus apricarius) in Schleswig-Holstein (links). Quelle: Winkler \& Klinge 2019. Die Lage des Untersuchungsgebiets in Schleswig-Holstein (rechts). Datengrundlage: ESRI; ๔ GeoBasis-DE/LVermGeo SH, ATKIS ${ }^{\circledR} B a s i s-$ DLM 2019, DTK100

15.1) überführt, daraus die besiedelten Teilflächen ermittelt und kartografisch dargestellt. Ausgangspunkt der Berechnung und Darstellung waren die minimalen Individuenzahlen der einzelnen Klassen. Die bei der Feldarbeit aufgenommenen Daten können eine geschätzte Ungenauigkeit von ca. 20\% aufweisen.
Die Daten der Erstaufnahme aus 1999 und 2000 liegen ausschließlich in aggregierter und kartografischer Form vor und wurden vom Verfasser ebenfalls mittels GIS aufbereitet. 

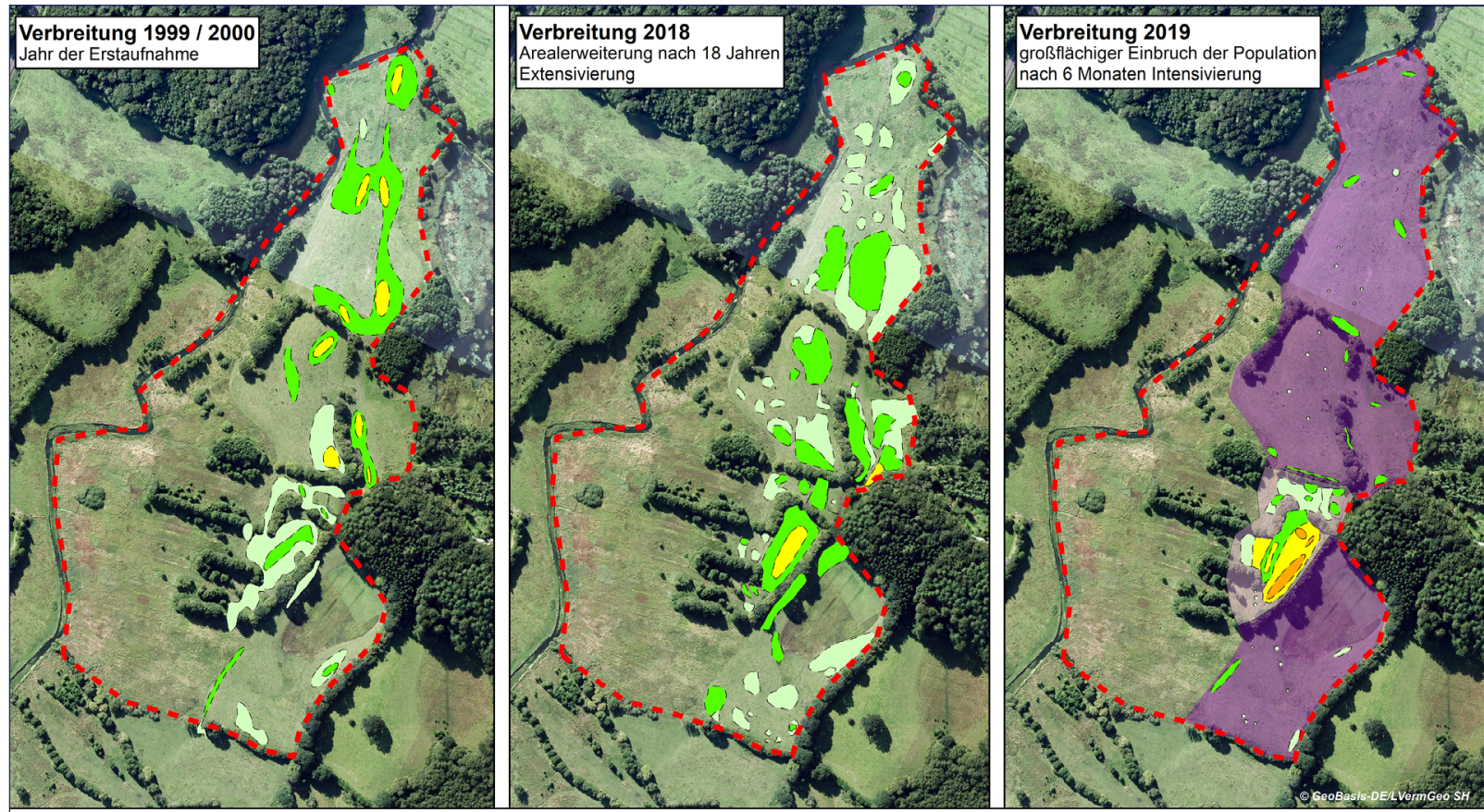

Wiederholungskartierung des Feldgrashüpfers (Chorthippus apricarius) im Oberen Eidertal bei Flintbek / Waldsiedlung 2018/2019 links im Vergleich: kombinierte Verbreitungskartierungen von 1999 und 2000

\begin{tabular}{|c|c|c|c|c|c|c|}
\hline \multirow{2}{*}{\multicolumn{2}{|c|}{ Geringe Dichte $(1-10$}} & \multirow{2}{*}{$\begin{array}{l}\text { iittlere Dichte }\left(11-50 \mathrm{o} / 100 \mathrm{~m}^{2}\right) \\
\text { ohe Dichte }\left(>50 \delta^{\prime \prime} / 100 \mathrm{~m}^{2}\right)\end{array}$} & Intensivierung 2019 & ㄴ. Kartiergebiet & \multirow[b]{2}{*}{100} & \multirow{2}{*}{400} \\
\hline & & & brachliegende Weidefläche 2019 & 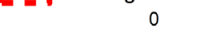 & & \\
\hline $\begin{array}{l}\text { Untersuchungen gefördert durch: } \\
\text { Stifung Naturschutz Schleswig- } \\
\text { Holstein }\end{array}$ & $\begin{array}{l}\text { Monitoring: } \\
\text { 1999: B. Holsten } \\
\text { 2000: B. Schulz } \\
\text { 2018, 2019: H. Nissen }\end{array}$ & $\begin{array}{l}\text { Bearbeiter: } \\
\text { Henning Nissen } \\
\text { Abteilung Landschaftsökologie, } \\
\text { Institut fur Naturu-und Ressourcenschutz } \\
\text { Christian-Albrechts-Universitut zu Kiel }\end{array}$ & $\begin{array}{l}\text { Datengrundlage: } \\
\text { OGeoBasis-DE/LVermGeo SH } \\
\text { DOP20 2017 } \\
\text { LVermGe SH, } 2017 \\
\text { Kartografe: H. Nissen }\end{array}$ & \begin{tabular}{|l|} 
Projekt: \\
Untersuchungen zur Bi \\
Invertebraten auf Fläch \\
Naturschutz Schleswig
\end{tabular} & $\begin{array}{l}\text { iversität von } \\
\text { der Stiftung } \\
\text { lstein }\end{array}$ & 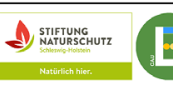 \\
\hline
\end{tabular}

Abb. 2: Vergleich der Verbreitung und Dichte des Feldgrashüpfers in unterschiedlichen Jahren.

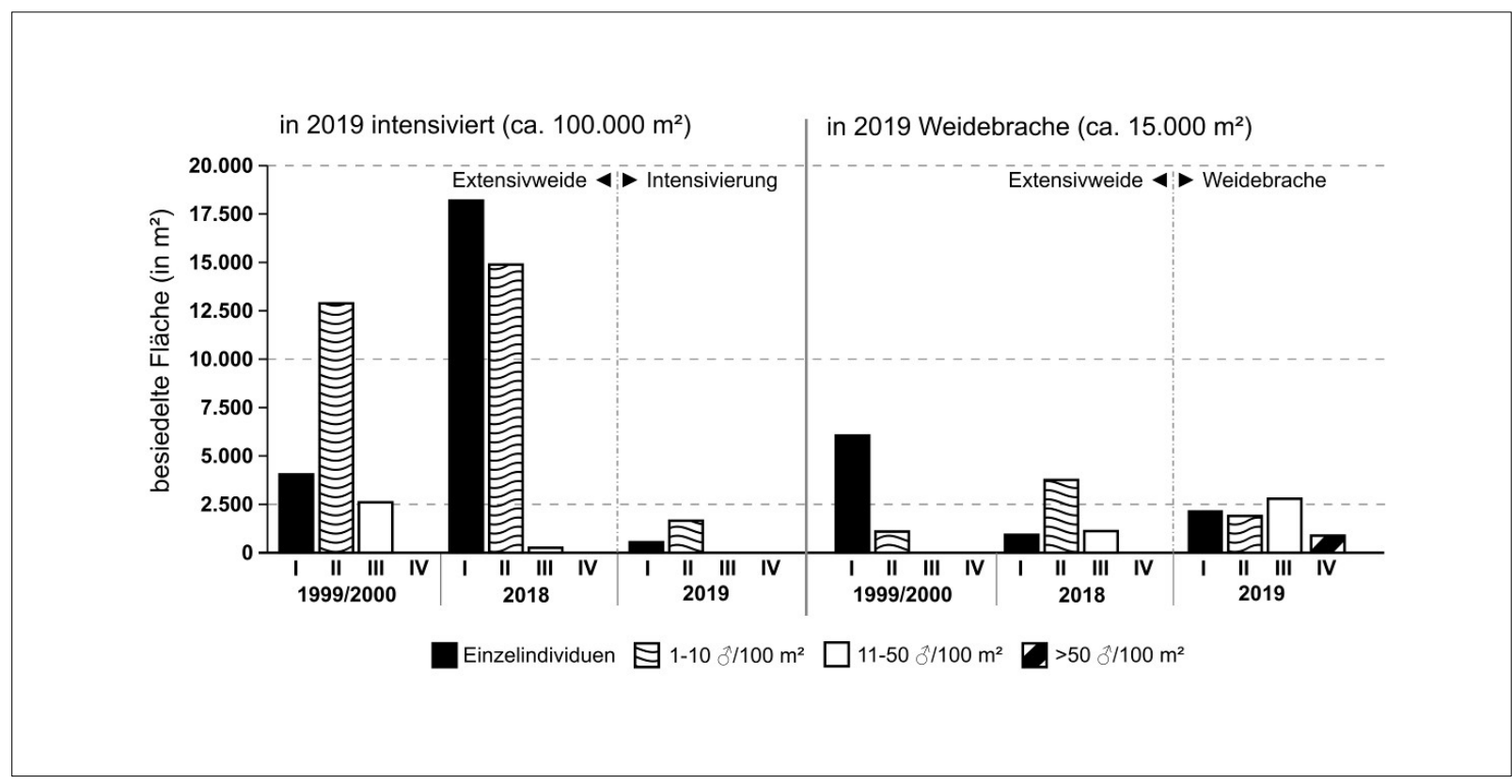

Abb. 3: Fluktuation der vom Feldgrashüpfer besiedelten Fläche der drei Erfassungszeiträume, aufgeteilt in Häufigkeitsklassen und unterteilt in die 2019 von der Nutzungsveränderung betroffenen Flächen. 
Tab. 2: Vom Feldgrashüpfer in den einzelnen Erfassungsjahren besiedelte Gesamtfläche (in $\mathrm{m}^{2}$ ). Erläuterung der Häufigkeitsklassen siehe Kap. 2.

\begin{tabular}{rrrr} 
Klasse & \multicolumn{2}{c}{ Jahr } & \\
& $\mathbf{1 9 9 9 / 2 0 0 0}$ & $\mathbf{2 0 1 8}$ & $\mathbf{2 0 1 9}$ \\
\hline I & 10.093 & 19.116 & 2.695 \\
\hline II & 13.974 & 18.641 & 3.552 \\
\hline III & 2.603 & 1.372 & 2.789 \\
\hline besiedelte Fläche & $\mathbf{2 6 . 6 7 1}$ & $\mathbf{3 9 . 1 3 0}$ & $\mathbf{9 . 9 1 8}$ \\
\hline
\end{tabular}

\section{Ergebnisse}

Bei der Ersterfassung im Jahr 1999/2000 von Holsten und Schulz handelte es sich bei den untersuchten Flächen z.T. noch um brachliegende Ackerflächen. Nachweise wurden damals ausschließlich auf den mineralischen Böden erbracht, hier war mit ca. 2,7 ha etwa 1/5 der untersuchten Gesamtfläche besiedelt. Nasse bis feuchte Bereiche wurden gemieden. Auf dem Großteil der besiedelten Fläche wurden geringe Dichten mit weniger als 10 Männchen je $100 \mathrm{~m}^{2}$ festgestellt. Mittlere Dichten (bis zu 50 Männchen / $100 \mathrm{~m}^{2}$ ) erstreckten sich auf wenige Flächen mit einer Größe von jeweils unter $600 \mathrm{~m}^{2}$. Solche Dichten fanden sich insbesondere in sonnenexponierten höherwüchsigen Grasfluren und grasigen Saumstrukturen entlang der Knicks, Feldgehölze und -hecken. Hohe Dichten konnten bei der Erstaufnahme nicht nachgewiesen werden.

Die Kartierung in 2018 erfolgte bei extremer Trockenheit. Weite Bereiche der Vegetation auf den mineralischen Böden waren, v.a. im Bereich der Kuppen, vertrocknet. Trotz dieser Extrembedingungen waren die bei der Erstkartierung noch vorhandenen Verbreitungslücken auf den mineralischen Böden mittlerweile geschlossen. Die besiedelte Fläche hatte sich von 2,7 ha auf 3,9 ha vergrößert. Die höchsten Dichten wurden in hochwüchsigen Gras- und Hochstaudenfluren erfasst, die von den Rindern nur mäßig verbissen wurden. Im Vergleich zur Erstkartierung nahmen die Flächen mit mittlerer Dichte etwa um die Hälfte ab. Auch die in 2018 nassen bis feuchten Teilflächen waren trotz extremer Trockenheit nicht besiedelt.
Die dortige Population ist im Jahr 2019 auf über 90\% ihrer im Vorjahr besiedelten Fläche verschwunden. Lediglich auf der Weidebrache besteht noch eine größere Teilpopulation. Hier konnte im Vergleich zu 2018 sogar ein Zuwachs sowohl in der Dichte als auch in der besiedelten Fläche verzeichnet werden. Erstmals bei der Untersuchung überhaupt konnten in diesem Teil vereinzelt hohe Dichten kartiert werden (Tab. 1).

\section{Diskussion}

Viele Heuschrecken haben in ihren Lebensstadien sehr unterschiedliche Lebensraumansprüche und benötigen von daher für ein langfristiges Überleben ein komplexes System einzelner geeigneter Mikrohabitate (Guido \& Gianelle 2001). Geht dieses System verloren, führt das zum Rückgang und Erlöschen lokaler Populationen, so auch beim Feldgrashüpfer (Reck 1998, Illich 2017, Woresch 2017). Trotz des auf europäischer Ebene ungefährdeten Bestands (Hochkirch et al. 2016) wird die Situation vieler lokaler Populationen und ihrer zunehmenden Gefährdung diskutiert (Reck 1998, Reck 2004, Maas et al. 2011, Illich 2017, Woresch 2017).

Der Feldgrashüpfer besiedelte vor der Extensivierung der untersuchten Flächen wahrscheinlich viele Saumstrukturen entlang von Ackerflächen und die bereits bestehenden Grünlandparzellen. Diese Vermutung basiert auf der von Reck (2004) herausgestellten Bedeutung von Ackersäumen für den Feldgrashüpfer in Süddeutschland. Die brachliegenden Äcker oder frisch eingesäten Flächen wurden vom Feldgrashüpfer großenteils noch nicht, bzw. nur in geringen Dichten besiedelt. Schulz (2003a) weist darauf hin, dass die Tiere zwar eine intensivere Beweidung und junge Brachen bevorzugen, aber bei homogener, kurzwüchsiger Pflanzendecke bei gleichzeitigem Fehlen von geeigneten Mikrohabitaten, wie Kratzdistel- oder o.ä. hochwüchsige Grashorste, Areale eher gemieden werden.

Die folgenden Populationsveränderungen werden ausschließlich in chronologischer Reihenfolge diskutiert. Die Verbreitungszunahme von 2000 nach 2018 kann nach Schulz (2003a) durch die Nettozunahme von Weideflächen mit hoher Habitateignung und einen funktionaleren und kleinräumigeren Lebensraumverbund im Zuge der fortschreitenden Entwicklung der extensiven Weidelandschaft ausgelöst worden sein. Das durch maximal 1,5 GVE beweidete Areal (Schulz 2003a) wies 2018 eine hohe Zahl an Mikrohabitaten auf. 
Mit 100 mm Niederschlag fielen von Mitte Mai bis zum 20. August 2018 etwas mehr als ein Drittel der für diesen Zeitraum durchschnittlichen Regenmengen (dwd. de, Station Kiel-Holtenau; Jahre 1986-2018). Dieser Zeitraum deckt den Schlupf der Larven (Reck 1998) bis zum Ende der Kartierung ab. Es wird vermutet, dass durch die extreme Trockenheit sogar weniger geeignete Flächen vorhanden waren und v.a. geringere Dichten aufgetreten sind als in Jahren mit den für die Region typischen Niederschlagsmengen. Ausschließlich in vergleichsweise stärker beschatteten Bereichen konnten die Tiere in mittleren Dichten erfasst werden. Viele Tiere mieden anscheinend die extrem trockenen Bereiche, die nur wenig Nahrung boten. Die Folgen der fortschreitenden Trockenheit können den Effekt der Intensivierung in 2019 durchaus verstärkt haben.

Detzel (1998) gibt an, dass die meisten adulten Heuschrecken bei intensiver Rinderbeweidung diese Flächen verlassen und in benachbarten unbeweideten Flächen zu finden sind. Die in 2019 extrem homogene Weide wurde vor der Beweidung flächendeckend gemäht, viele Meter Knick wurden auf den Stock gesetzt, was sicherlich zum Verlust der für den Feldgrashüpfer wichtigen Mikrohabitate führte. Da der Feldgrashüpfer seine Eier in den Boden legt (Reck 1998), kann es sein, dass trotz Nutzungsintensivierung noch viele Individuen ab Mai/April auf den beweideten Arealen geschlüpft sind. In 2018 wurden viele Eipakete in dem Jahr noch in geeigneten Lebensräumen abgelegt, die dann in 2019 an Stellen schlüpften, die nunmehr ungeeignet waren. Viele überlebende Individuen könnten also wegen der nicht mehr vorhandenen Habitateignung auf die nicht beweideten Flächen, insbesondere die brachliegende Weide abgewandert sein. Reck (1998) beschreibt die Art als ortstreu und migrationsschwach, aber die physische Ausbreitungskraft insbesondere die der Larven und die der Männchen sei höher als das von Reck beobachtete Verhalten. Schulz (2003a) konnte beobachten, dass die Art in jungen Brachestadien verbreitet war. Die 2019 junge, brachliegende Fläche wies ein hohes Maß an Mikrohabitatstrukturen auf. Niedrigwüchsige Grasfluren und hochwüchsigere Hochstauden entlang von Feldgehölzen und Knicks wurden erstmals in hohen Dichten besiedelt. Schulz (2003a) und Buchweitz et al. (1990) stellen dieses Mosaik als bevorzugte Bereiche heraus. Für einen langfristigen Erhalt der lokalen Population ist diese Fläche vermutlich zu kleindimensioniert und könnte mit fortschreitender Brachedauer, bzw. Wiederaufnahme ins Weideregime ihre positive Habitateignung verlieren.
Die Intensivierung und Parzellierung des kleinen Teilbereichs der ehemaligen Großweidelandschaft könnte für die lokale Feldgrashüpferpopulation bestandsgefährdend sein. Während ein Großteil der untersuchten Habitatflächen aktuell nicht mehr vorhanden und geeignete Lebensräume wieder fragmentiert worden sind, war der Bestandsrückgang auf diesen Flächen die vermutete Konsequenz. Auch die kurzfristige hohe Habitateignung der Brachefläche kann durch Nutzungintensivierung abrupt, oder durch langfrisitge Verbrachung wieder abnehmen.

Die Nutzungsentwicklung der anderen, nicht untersuchten Teilbereiche der Großweidelandschaft sind ähnlich (Winkler, schr. Mittlg.). Bereiche mit derzeit hoher Habitateignung (i. d. R. derzeitige Brachen) können ebenfalls ihre hohe Eignung verlieren. Es wird vermutet, dass so in der gesamten Großweidelandschaft starke Bestandsrückgänge zu verzeichnen sind, die im Extremfall zum Erlöschen der Lokalpopulation führen könnte. Das ehemals resiliente Großweidesystem hat viele der Ausweichräume verloren, die bei hoher Dynamik die beschriebenen Fluktuationen abpuffern können. Um die Auswirkungen der Intensivierung $\mathrm{zu}$ beurteilen, könnte eine erneute Untersuchung der Folgegeneration in 2020 wichtige Erkenntnisse liefern.

Insgesamt zeigt diese Untersuchung, warum die Intensivierung insbesondere der landwirtschaftlichen Flächennutzung mit der damit einhergehenden Beseitigung von Habitatvielfalt, Verbrachung von Grenzertragsstandorten, Verlust von Saumstrukturen und Mikrohabitaten (Emmerson et al. 2016, Gossner et al. 2016) eine der gravierendsten Ursachen für den Verlust von Biodiversität insgesamt ist (Vgl. u.a. Hallmann et al. 2017, Seibold et al. 2019). Das Fehlen von extensiven Nutzungsformen auf lokaler Ebene kann den Austausch von Teilpopulationen und die (Wieder-)Besiedlung verwaister, aber geeigneter Lebensräume erheblich negativ beeinflussen. In diesem Fall ist eine über 20 Jahre laufende naturschutzfachlich begründete Entwicklung innerhalb kürzester Zeit wieder rückgängig gemacht worden und lässt den Schluss zu, dass dauerhafte Maßnahmen gegenüber zeitlich befristeten Maßnahmen sofern möglich bevorzugt werden sollten (Schöttker \& Wätzold 2018).

Selbst Teilpopulationen einer ansonsten weit verbreiteten und (noch) relativ häufigen und von (extensiver landwirtschaftlicher) Landnutzung profitierenden Art können demnach durch zu starke Intensivierung der Landnutzung innerhalb kürzester Zeit massiv einbrechen. Die Umsetzung einer (Intensivierungs-)Maß- 
nahme, wie z. B. die Knickpflege oder eine einschürige Mahd vor der Beweidung von Grünland hat vermutlich einen zwar messbaren, aber geringeren Effekt auf die Bestände dieser oder ähnlicher Arten. Das Zusammenwirken aller auf den Flächen umgesetzten Maßnahmen (Verlust von Kernlebensräumen, Mikrohabitaten und Saumstrukturen in Kombination mit der Fragmentierung ehemals verbundener Habitate) wird hier als ausschlaggebend eingeschätzt. Der dabei beobachtete Verlust von über 90 \% der Fläche/Biomasse dieser Art im intensivierten Areal ist vergleichbar mit den von Seibold et al. (2019) im Grünland und Hallmann et al. (2017) in Schutzgebieten herausgestellten Rückgängen anderer Arthropoden. Der generell zu beobachtende Biomasseverlust der Insekten ist demnach auf lokaler Ebene nicht unbedingt ein schleichender Prozess, sondern kann klar auf bestimmte Ursachen zurückgeführt werden.

Betont werden muss, dass Heuschrecken wie der hier betrachtete Feldgrashüpfer, im extensiv genutzten Grünland besonders häufig, teils sogar in Massen auftreten können und einen erheblichen Anteil der tierischen, epigäischen Biomasse darstellen, wodurch sie ein besonders wichtiges Element in der Nahrungskette sein können (Belovski \& Slade 2000, Hawlena \& Schmitz 2010, Zhu et al. 2017, Zuna-Kratky et al. 2017). Unter den Vertretern in Deutschland gibt es trotz dieser hohen Bedeutung, wie bei vielen anderen Insektengruppen, keine nach FFH-Richtlinie geschützte Art. Auch deshalb werden die für Grünlandlebensräume sehr prägenden Arten derzeit kaum berücksichtigt. Selbst vor wenigen Jahren noch häufige Arten verschwinden derzeit sukzessive aus der Landschaft, wie das Beispiel des Sumpfgrashüpfers zeigt (Rohde et al. 2017) und wie es hier für den Feldgrashüpfer auf zumindest lokalem Maßstab gezeigt werden konnte.

\section{Dank}

Ich danke Björn Schulz für wichtige Hinweise während der Untersuchung und Anmerkungen zum Manuskript. Bei dem Flächeneigentümer bedanke ich mich für die Betretungsgestattung seiner Flächen, Lisa Bönke von der Stiftung Naturschutz Schleswig-Holstein sei für die Vermittlung der Flächenbetretung gedankt. Bei der Stiftung Naturschutz Schleswig-Holstein bedanke ich mich für die finanzielle Förderung des Vorhabens.

\section{Autor}

\section{Henning Nissen ${ }^{(1)}$}

Henning Nissen ist wissenschaftlicher Mitarbeiter am Institut für Natur- und Ressourcenschutz in Kiel. Seine Arbeitsgebiete umfassen die Wiedervernetzung von Lebensräumen und entomofaunistische Forschungsansätze zur Überwindung artifizieller Barrieren.

\section{Literatur}

Belovsky GE, Slade, JB (2000) Insect herbivory accelerates nutrient cycling and increases plant production. PNAS 97(26):14412-14417.

Buchweitz M, Detzel P, Hermann G (1990) Zur Bedeutung von Feldrainen als Lebensraum für Chorthippus apricarius (L. 1758) (Orthoptera, Saltatoria, Acrididae). Articulata 5(2):49-58.

Detzel P (1998) Die Heuschrecken Baden-Württembergs. Verlag Eugen Ulmer, Stuttgart.

Emmerson M, Morales MB, Oñate JJ, Batáry P, Berendse F, Liira J, Aavik T, Guerrero I, Bommarco R, Eggers S, Pärt T, Tscharntke T, Weisser W, Clement $L$, Bengtsson J (2016) How agricultural intensification affects biodiversity and ecosystem services. Adv. Ecol. Res. 55:43-97.

Gossner MM, Lewinsohn TM, Kahl T, Grassein F, Boch S, Prati D, Birkhofer K, Renner SC, Sikorski J, Wubet T, Arndt H, Baumgartner V, Blaser S, Blüthgen N, Börschig C, Buscot $F$, Diekötter T, Jorge LR, Jung K, Keyel $A C$, Klein A-M, Klemmer $S$, Krauss J, Lange $M$, Müller J, Overmann $J$, Pašalić E, Penone C, Perović DJ, Purschke 0 , Schall P, Socher SA, Sonnemann I, Tschapka M, Tscharntke T, Türke M, Venter PC, Weiner CN, Werner M, Wolters V, Wurst S, Westphal C, Fischer M, Weisser WW, Allan E (2016) Landuse intensification causes multitrophic homogenization of grassland communities. Nature 540:266-269.

Guido M, Gianelle D (2001) Distribution patterns of four Orthoptera species in relation to microhabitat heterogeneity in an ecotonal area. Acta Oecologica 22:175-185.

Hallmann CA, Sorg M, Jongejans E, Siepe $\mathrm{HH}$, Hofland N, Schwan H, Stenmans W, Müller A, Sumser H, Hörren T, Goulson D, Kroon H (2017) More than 75 percent decline over 27 years in total flying insect biomass in protected areas. PLOS ONE 12(10):1-21.

Hawlena D, Schmitz OJ (2010) Herbivore physiological response to predation risk and implications for ecosystem nutrient dynamics. PNAS 107(35):15503-15507.

Hochkirch A, Nieto A, García Criado M, Cálix M, Braud Y, Buzzetti FM, Chobanov $D$, Odé $B$, Presa Asensio JJ, Willemse L, Zuna- Kratky T, Barranco Vega $P$, Bushell M, Clemente ME, Correas JR, Dusoulier F, Ferreira $S$, Fontana $P$, García MD, Heller K-G, lorgu IȘ, Ivković $S$, Kati V, Kleukers

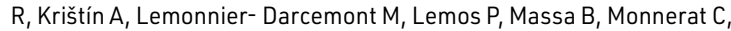
Papapavlou KP, Prunier F, Pushkar T, Roesti C, Rutschmann F, Șirin D, Skejo J, Szövényi G, Tzirkalli E, Vedenina V, Barat Domenech J, Barros F, Cordero Tapia PJ, Defaut B, Fartmann T, Gomboc $S$, Gutiérrez-Rodríguez J, Holuša J, Illich I, Karjalainen S, Kočárek P, Korsunovskaya O, Liana A, López H, Morin D, Olmo-Vidal JM, Puskás G, Savitsky V, Stalling T, Tumbrinck J (2016) European Red List of Grasshoppers, Crickets and Bush-crickets. Publications Office of the European Union, Luxembourg.

Illich I (2017) Feldgrashüpfer Chorthippus apricarius (Linnaeus, 1758). Denisia 39:747-751.

Jensen K, Granke 0, Hoppe B, Kieckbusch J, Trepel M, Leiner U(2001) Weidelandschaft Eidertal - Naturschutz durch extensive Beweidung und Wiedervernässung. Petermanns Geografische Mitteilungen 145:38-49 
Köhler G (2001) Fauna der Heuschrecken (Ensifera und Caelifera) des Freistaates Thüringen. Thüringer Landesanstalt für Umwelt, Abt. Ökologie und Naturschutz, Jena.

Maas S, Detzel P, Staudt A (2002) Gefährdungsanalyse der Heuschrecken Deutschlands. Verbreitungsatlas, Gefährdungseinstufung und Schutzkonzepte:Ergebnisse aus dem F+E-Vorhaben 89886015 des Bundesamtes für Naturschutz. Landwirtschaftsverlag, Münster.

Maas S, Detzel P, Staudt A (2011) Rote Liste und Gesamtartenliste der Heuschrecken (Saltatoria) in Deutschland. In:Binot-Hafke M et. al (Hg.) Rote Liste gefährdeter Tiere, Pflanzen und Pilze Deutschlands, Bd. 3:Wirbellose Tiere (Teil 1). Landwirtschaftsverlag, Münster, S. 577-606.

Reck H (1993) Haben Tierbauten eine Bedeutung als Habitatbaustein für den Feldgrashüpfer (Chorthippus apricarius L 1758)? Articulata 8 (1):45-51.

Reck H (1998) Chorthippus apricarius. In:Detzel P (Vf.) Die Heuschrecken Baden-Württembergs. Verlag Eugen Ulmer, Stuttgart, S. 470-479.

Reck H (2004) Schutz für den in Baden-Württemberg vom Aussterben bedrohten Feldgrashüpfer (Chorthippus apricarius L) - Teil 1. Naturschutz Alb-Neckar 1:2-19.

Rohde K, Hau Y, Kranz N, Weinberger J, Elle O, Hochkirch A (2017) Climatic effects on population declines of a rare wetland species and the role of spatial and temporal isolation as barriers to hybridization. Funct. Ecol. $31(6): 1-13$.

Seibold S, Gossner MM, Simons NK, Blüthgen N, Müller J, Ambarli D, Ammer $C$, Bauhus J, Fischer M, Habel JC, Linsenmair KE, Nauss T, Penone C, Prati D, Schall P, Schulze ED, Vogt J, Wöllauer S, Weisser WW (2019) Arthropod decline in grasslands and forests associated with landscapelevel. Nature 574:671-691.

Schöttker 0, Wätzold F (2018) Landkauf oder Kompensation von Landwirtinnen und Landwirten? Ökonomische Analyse von Naturschutmaßnahmen zur Erhaltung eines oligotrophen Sees. Natur- und Landschaft 11(93):494-500.

Schulz B (2003a) Die Reaktion ausgewählter Tierartengruppen (Coleoptera:Carabidae und Orthoptera:Acrididae) auf eine großflächige Extensivbeweidung am Beispiel der Weidelandschaft Oberes Eidertal. Dissertation, Agrar- und Ernährungswissenschaftliche Fakultät, Christian-Albrechts-Universität zu Kiel, Deutschland.

Schulz B (2003b) Zur Bedeutung von Beweidung und Störstellen für Tierarten am Beispiel der Verteilung von Feldheuschreckengelegen im Grünland. Articulata 18(2):151-178.

Voith J, Beckmann A, Sachteleben J, Schlumprecht H, Waeber G (2016) Rote Liste und Gesamtartenliste der Heuschrecken (Saltatoria) Bayerns. Bayerisches Landesamt für Umwelt (LfU), Augsburg, urn:nbn:de:bvb:12-babs2-0000161242.

Winkler C (1999) Entwicklung von Strategien für den Heuschreckenschutz in Schleswig-Holstein. Diplomarbeit, Christian-Albrechts-Universität zu Kiel (unveröffentlichtes Manuskript).

Winkler C, Klinge, A (2019) Die Heuschrecken Schleswig-Holsteins. Arbeitsatlas 2019. Faunistisch-Ökologische Arbeitsgemeinschaft e.V., Kiel.

Winkler C, Haacks M (2019) Die Heuschrecken Schleswig-Holsteins. Rote Liste. Landesamt für Landwirtschaft, Umwelt und ländliche Räume des Landes Schleswig-Holstein, Flintbek.

Worschech K (2017) Vorkommen des Feldgrashüpfers Chorthippus apricarius (Linnaeus, 1758) (Saltatoria:Acrididae) in der intensiv genutzten Agrarlandschaft des Altenburger Landes (Thüringen). Mauritania 32:360-386

Zhu H, Qu Y, Zhang D, Li J, Wen M, Wang D, Ren B (2017) Impacts of grazing intensity and increased precipitationon of a grasshopper assemblage (Orthoptera:Acrididae) in a meadow steppe. Ecological Entomology 42:458-468.

\section{Open Access}

>> Der Artikel ist unter der Creative-Commons-Lizenz Namensnennung 4.0 International veröffentlicht. Den Vertragstext finden Sie unter:https://creativecommons.org/licenses/by/4.0/deed.de. Bitte beachten Sie, dass einzelne, entsprechend gekennzeichnete Teile des Artikels von der genannten Lizenz ausgenommen sein bzw. anderen urheberrechtlichen Bedingungen unterliegen können. 